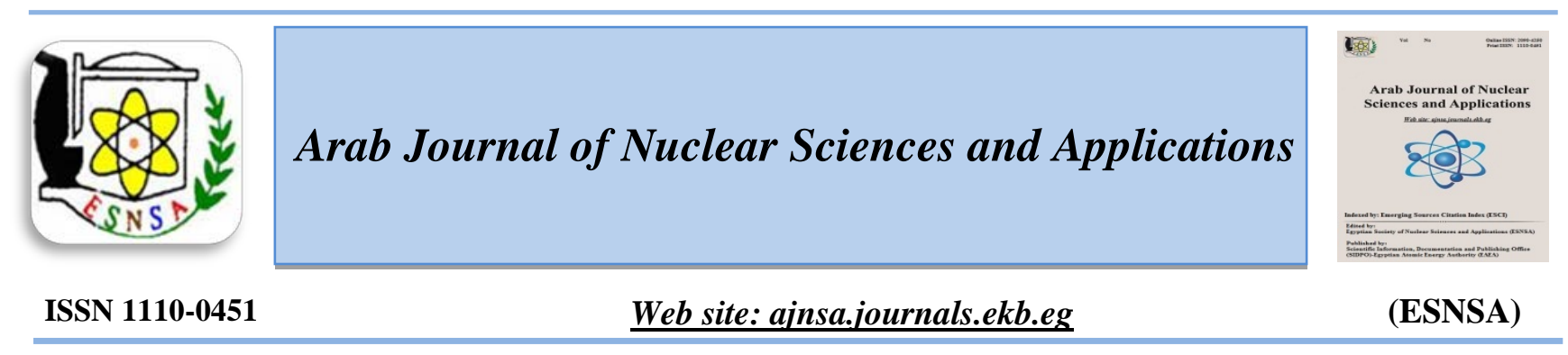

\title{
Experimental Work for Minimizing Transmission Power Losses Using Nanotechnology
}

\author{
Amal F. Abdel Gawad, Mohamed Taalab, A. ElSebaei, and N. S. Ali* \\ Faculty of Engineering, Zagazig, Egypt
}

\begin{abstract}
Received $2^{\text {nd }}$ Aug 2017 The present study illustrates a new application of nanotechnology in electrical power transmission Accepted $18^{\text {th }}$ Sep 2017 involving the coating the transmission lines of type ACSR with a nano layer of copper to minimize the resistance of the transmission and so minimize the transmitted power losses. The substrate used in the present investigation is made of aluminium of purity $99.9 \%$. The coating process is done by Hybrid Physical Vapor Decomposition using magnetron sputtering technique. The experimental testing has shown that the resistance of the coated substrates is reduced and the obtained results have proven the desired goal of power loss reduction in transmission lines.
\end{abstract}

Keywords: Nanotechnology, Power losses, Magnetron sputtering, Nanoparticles, Electrical resistance

\section{Introduction}

The huge amount of power generated in a power station (hundreds of MW) is to be transported over a long distance (hundreds of kilometers) to load centers to cater power to consumers with the help of transmission line and transmission towers. The important parameters in the design and operation of a transmission line include; the determination of voltage drop, line losses and efficiency of transmission. These values are greatly influenced by the line constants $\mathrm{R}, \mathrm{L}, \mathrm{C}$ of the transmission line. For instance, the voltage drop in the line depends upon the values of above three line constants. Similarly, the resistance of the transmission line conductor is the most important cause of power loss in the line and determines the transmission efficiency.

ACSR is one of the most used conductors in transmission lines Figure (1). It consists of alternate layers of stranded conductors, spiraled in opposite directions to hold the strands together, surrounding a core of steel strands. The number of steel strands may be 7 or 19 cables. The purpose of introducing a steel core inside the stranded aluminum conductors is to obtain a high strengthto-weight ratio. A stranded conductor offers more flexibility and is easier to manufacture than a solid large conductor. However, the total resistance is increased because the outside strands are larger than the inside strands on account of the spiraling. If the $\mathrm{Al}$ and Steel strands are separated by paper then this kind of ACSR is used in EHV lines and called expanded ACSR.

Nanotechnology introduces promising acceptable solutions and products to achieve reliable sustainable environment. The term nanotechnology embraces many different fields and specialties, including engineering with bringing existing technologies down to a very small scale, measured in nanometers. Processes and functionality take place at the nanoscale, exhibiting properties not available in the bulk material. At this size, atoms and molecules work differently, and provide a variety of surprising and interesting uses.

Corresponding Author: nehadsalah@yahoo.com

DOI: 10.21608/ajnsa.2018.6514

(C) Scientific Information, Documentation and Publishing Office (SIDPO)-EAEA 
A review on applications of Nanotechnology in the energy sectors including the transmission system was presented in a previous study [1]. Many researches were devoted to improve the performance of transmission lines in power systems applying various ideas and techniques as will be illustrated.

One of these ideas was reported by Kwang et al. [2] through the use of eight Polymer nanocomposites reinforced with multi-walled carbon nanotubes (MWCNTs) for Semiconducting layers of high-voltage electrical power cables. The mechanical, thermal and electrical properties were investigated as a function of polymer type. The dependence of strain on the volume resistance was also studied. The nanocomposite (EEA/MWCNT) [ethylene-co-(ethyl acrylate)]/MWCNT showed the best combination of tensile strength, elongation at break and Young's modulus compared with a commercial composite. Based on the criteria of tensile properties and volume resistance, a poly (EEA/MWCNT) nano composite was selected as the best candidate for the semiconducting layers of high-voltage electrical power cables.

Networks of CNTs were used to build planar transmission lines which functioned as strip line waveguides in the frequency range studied by Mahmoud et al [3] who replaced conventional metallic conductors in electric circuits by CNTs, in general, and RF/microwave circuits in particular. From results, the losses of CNT networks were still high compared to copper. This was due to the low-cost CNTs used, with 1:3 metallic to dielectric composition. As fabrication techniques improved, it was anticipated that materials consisting of only metallic CNTs would be produced at low cost.

The fabrication of iodine-doped, double-walled nanotube cables having electrical resistivity reaching $10^{-7} \Omega$.m was reported by Yao et al [4]. Due to the low density, their specific conductivity (conductivity/weight) had been higher than copper and aluminium and was only just below that of the highest specific conductivity metal, sodium. The application of such nanotube cables was demonstrated by partly replacing metal wires in a household light bulb circuit. The conductivity variation as a function of temperature for the cables was five times smaller than that for copper.
A study was recently made [5] aiming to increase stability and maximum loadability of a system by replacing congestion conventional transmission line with new carbon nano tube (CNT) line. In study, the authors focused on reducing the congestion of the system and /or system losses, hence maximum loading point of the system increased and system dynamic performance improved. Based on simulation results could conclude that (CNT) line instead of congested line gave higher maximum loadability and minimized the worst case voltage deviations. Also (CNT) line improved system dynamic stability performance through time domain simulation results.

Copper was used as a coating metal in the investigation reported by Minh-Tung et al. [6] since electrical resistivity of $\mathrm{Cu}$ films had been a very important factor for using interconnects. Copper is an attractive interconnecting material due to its low resistivity and super resistance to electro-migration. Thin films of $\mathrm{Cu}$ are deposited by various techniques such as evaporation, sputtering, chemical vapour deposition electroplating, and ion beam deposition. Among these techniques, direct current (DC) magnetron sputtering is one of the best methods for practical preparation of $\mathrm{Cu}$ films. Since DC magnetron sputtering technique has the advantage of much better productivity than other deposition methods, it is widely used as mass production processes.

Arun Augustin et al. [7] applied a copper coated touch surface by DC magnetron sputtering on zincated and non-zincated aluminium substrate. They studied the difference between sputtering of copper on two different aluminium substrates. XRD characterization indicated that by zincating, the tendency for copper alignment could be reduced. SEM studies indicated that the copper crystallites on the zincated substrate were predominantly in the range of $125-200 \mathrm{~nm}$ whereas in the case of non-zincated substrate, it was in the range of 40-60 nm. Contact angle studies showed that the wettability of copper coated on nonzincated $\mathrm{Al}$ was more compared to copper coated on zincated aluminium.

The objectives of this paper are: (i) develop new advanced planar transmission line (TL), (ii) characterize its electrical properties. To achieve this goal, $\mathrm{Cu}$ thin films are deposited on 
aluminium alloy substrates using magnetron sputtering source (MS) in Hybrid Physical Vapor Deposition and plasma enhanced chemical vapor deposition (Hybrid PVD-PECVD) equipment. The effect of different sputtering powers and deposition times on the electrical properties is investigated to get acceptable results in minimizing the transmission losses.

\section{Experimental Setup}

Evaporation of metal using MS source in Hybrid PVD - PECVD for the formation of copper coating is so far known as a high-efficiency laboratory technique. The equipment of Hybrid PVD PECVD (protec nanoflex 400) are shown in Figure (2).

The aluminum alloy (Al) used for this experiment(fig. 3) is of purity $99.5 \%$ with some other metals such as Fe (0.372\%), Ti (0.021\%), Si $(0.064 \%)$, and others with very small ratios. The alloy was made by Spectromax- Chemical Composition of metals. The dimension of this substrate alloy is $5 \mathrm{~cm} * 6 \mathrm{~cm} * 0.5 \mathrm{~cm}$. The $\mathrm{Al}$ substrates are cleaned in diluted isopropanol solution for 1 minute, and are rinsed in de-ionized water prior to be loaded into a vacuum chamber. Normally the first step to execute is the "vacuum and heating" phase inside the chamber filled with the substrates, the high vacuum must to be created

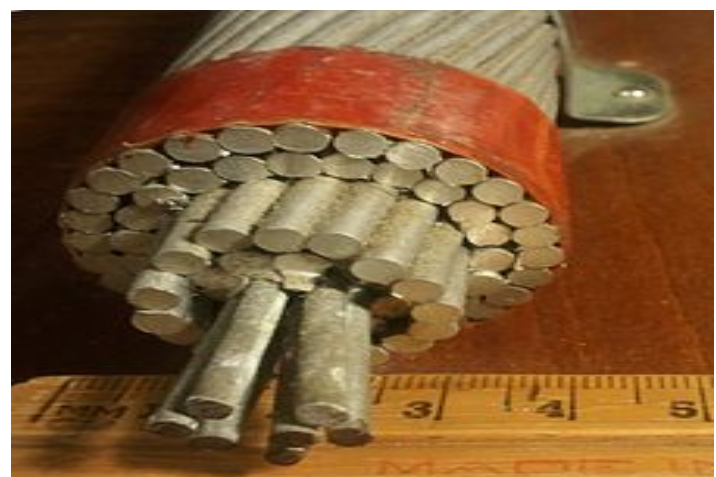

Fig. (1): Aluminum Cored Steel Reinforced (ACSR) to eliminate as much as possible the residual atmosphere. The deposition chamber is evacuated to $10^{-5} \mathrm{~Pa}$ base pressure. In the deposition chamber the vacuum level for optimal coating cycle is created by a turbo molecular pump mounted above the lid of the chamber. Before the deposition of $\mathrm{Cu}$ films, a $\mathrm{Cu}$ target $(99.999 \%$ purity and $400 \mathrm{~mm}$ *66 mm*8 mm thickness) was pre-suttered in pure argon for 10 minute in order to remove oxide layer on the surface of the target. $\mathrm{Cu}$ films are deposited by MS in pure argon gas at $6 * 10^{-1} \mathrm{~Pa}$. Chamber temperature is kept constant at $100{ }^{\circ} \mathrm{C}$. The distance from the $\mathrm{Cu}$ target to $\mathrm{Al}$ substrate in the experimental is $8 \mathrm{~cm}$. Different values of applied electrical power are used (1.05 KW, $1.5 \mathrm{KW}$, and 2 $\mathrm{KW}$ ).

The magnetron sputtering technique is applied on this alloy in order to coat it with a nano layer of copper for different periods of time ( $1 / 2$ hour and one hour). This experiment has been done under temperature of $100 \mathrm{C}^{\circ}$. Different values of applied electrical power are used $(1.05 \mathrm{KW}, 1.5 \mathrm{KW}$, and 2 $\mathrm{KW}$. The thickness of copper layer for each case is illustrated by (Stylus Profilmeter model- Dektak 150). These thickness measurements are the input data to resistance measurement device (KEYSIGHT B1500A Semiconductor Device Analyzer) in order to measure their resistance.

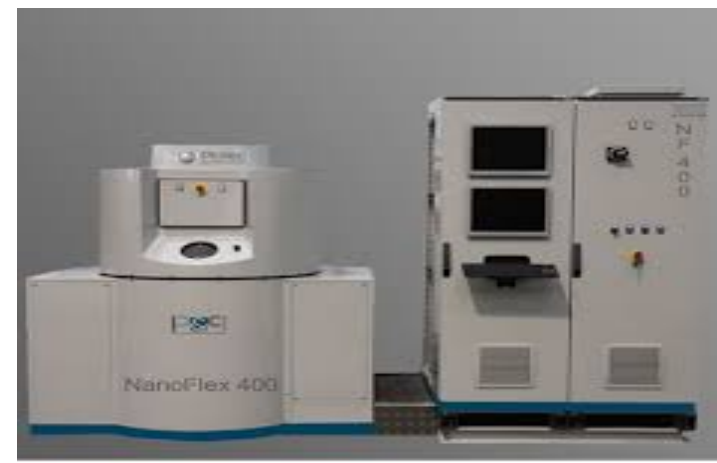

Fig. (2): Hybrid PVD - PECVD device

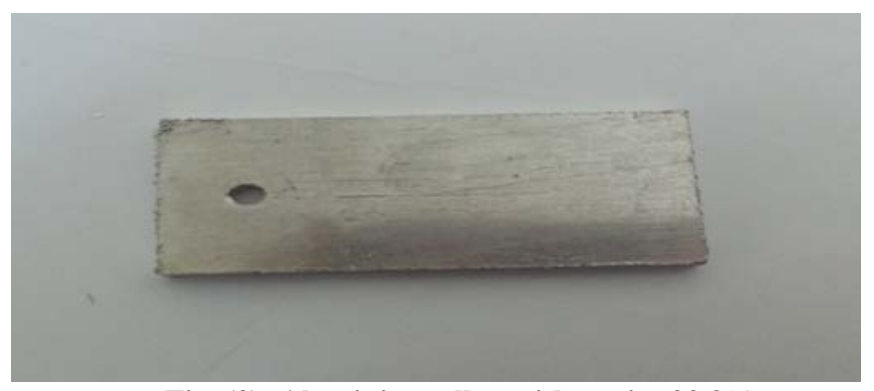

Fig. (3): Aluminium alloy with purity $99.9 \%$ 


\section{Results and Discussion}

The coated substrate with nano particles of copper for one case of the test cases is shown in Figure (4). Scanning Electron Micrographs (SEM) of Nano copper coatings for the sample that has been applied to $100 \mathrm{c}^{\circ}, 1$ hour, and $2 \mathrm{KW}$ power resulted in the photo is shown in Figure (5).

Figure (5) shows the scanning electron microscopic view of the top surface of the copper coating. The topographic image shows that coating is formed by very fine clusters of crystallites. The histogram for size distribution of clusters of crystallites showed indicates that major clusters are in between 83.08-94.99 $\mathrm{nm}$ size.

The parameters used in the coating process with magnetron sputtering source, thickness of the resulted coating layers, and the resistances of the coated substrates (AL-Cu) are presented in Table (1) as well as in Figures (5 to 8).

From the curve in Figure (6), it can be deduced that, the thickness of the coating layer increases as the applied power to the Hybrid PVD-PECVD

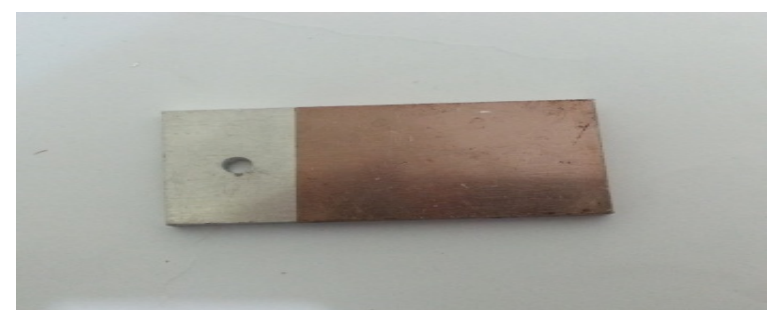

Fig. (4): coated substrate

Table (1): Results of the coating process

\begin{tabular}{ccccc}
\hline $\begin{array}{c}\text { Substrate } \\
\text { NO. }\end{array}$ & $\begin{array}{c}\text { Power } \\
(\text { KW })\end{array}$ & $\begin{array}{c}\text { Time } \\
(\mathrm{hr})\end{array}$ & Thickness & $\begin{array}{c}\text { Resistance } \\
(\Omega)\end{array}$ \\
\hline 1 & - & - & $0.5 \mathrm{~cm}$ & 0.5 \\
2 & 1.05 & $1 / 2$ & $569.75 \mathrm{~nm}$ & 0.43 \\
3 & 1.05 & 1 & $1030.33 \mathrm{~nm}$ & 0.38 \\
4 & 1.5 & $1 / 2$ & $587.8 \mathrm{~nm}$ & 0.42 \\
5 & 1.5 & 1 & $1101 \mathrm{~nm}$ & 0.34 \\
6 & 2 & $1 / 2$ & $682.02 \mathrm{~nm}$ & 0.40 \\
7 & 2 & 1 & $1221.34 \mathrm{~nm}$ & 0.33 \\
\hline
\end{tabular}

device increases at any time. This is because the crystallite size increases with the sputtering power since the higher surface mobility caused by high adatom energy strengthens the crystallinity of the $\mathrm{Cu}$ films at higher sputtering power.

Since the thickness has been increased by increasing the applied power, the resistance to the electrical current will by decreased according to the equation;

$R=\rho \frac{l}{A} \quad \Omega$

Where; $\mathrm{R}$ : is the resistance of the material, $\rho$ : is the resistivity of the material, $\mathrm{l}$ : is the material length, $\mathrm{A}$ : is the area of the material.

This is clearly appeared in Figure (7). By increasing the applied power for the coating operation, the resistance of the substrate decreases.

As obvious in Figures (8 and 9), by increasing the time of coating operation, the thickness increases and the resistance decreases. This assures equation 1.

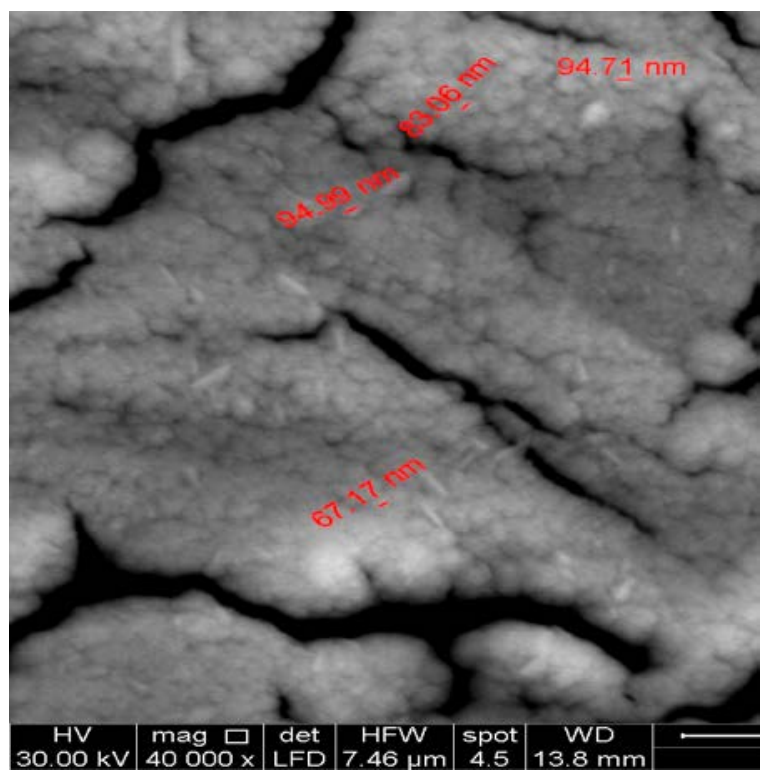

Fig. (5): SEM photo for the coating layer on the substrate 


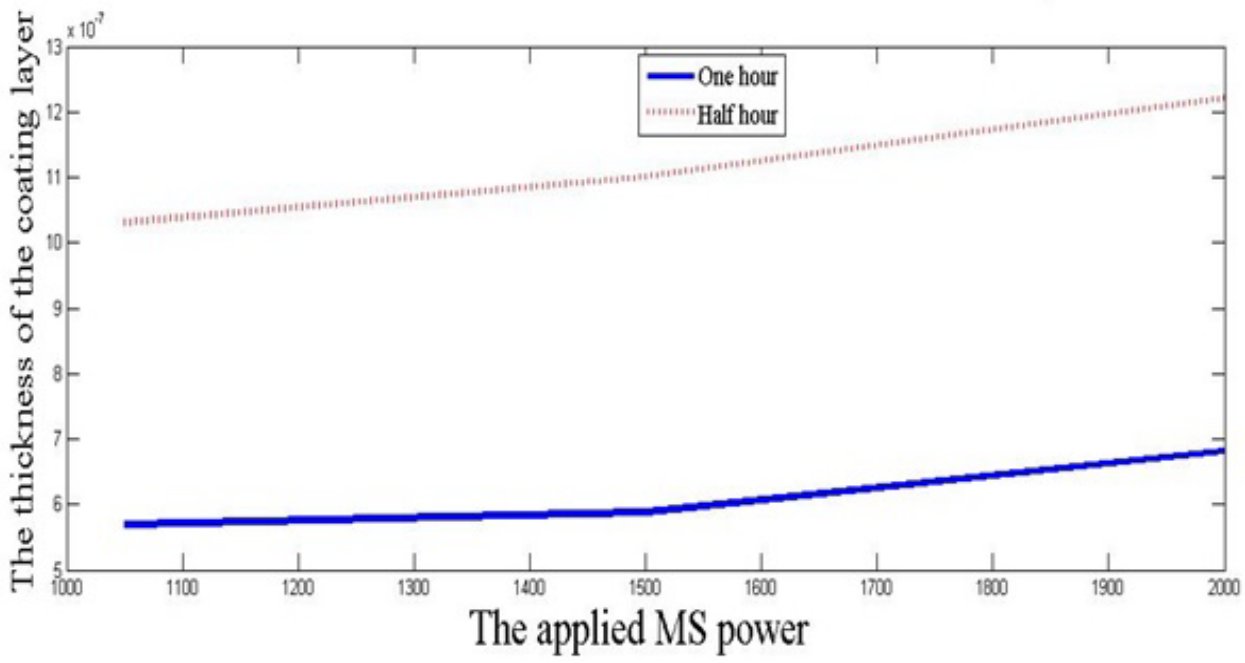

Fig. (6): The effect of MS power on the thickness of the coating layer

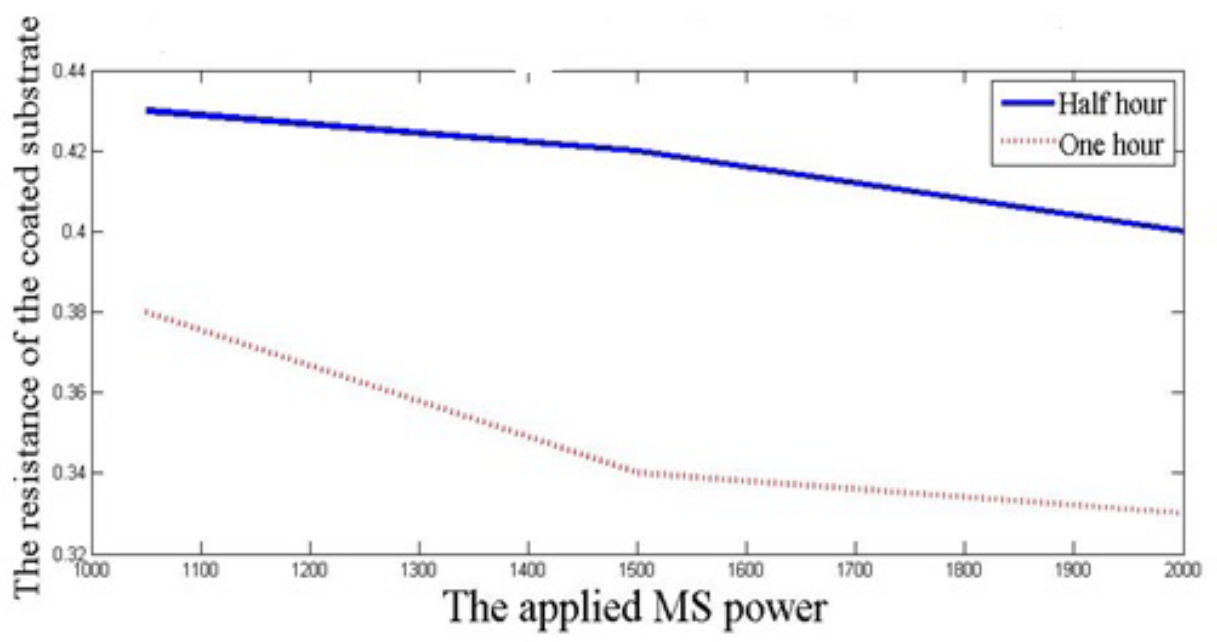

Fig. (7): The effect of MS power on the resistance of substrate

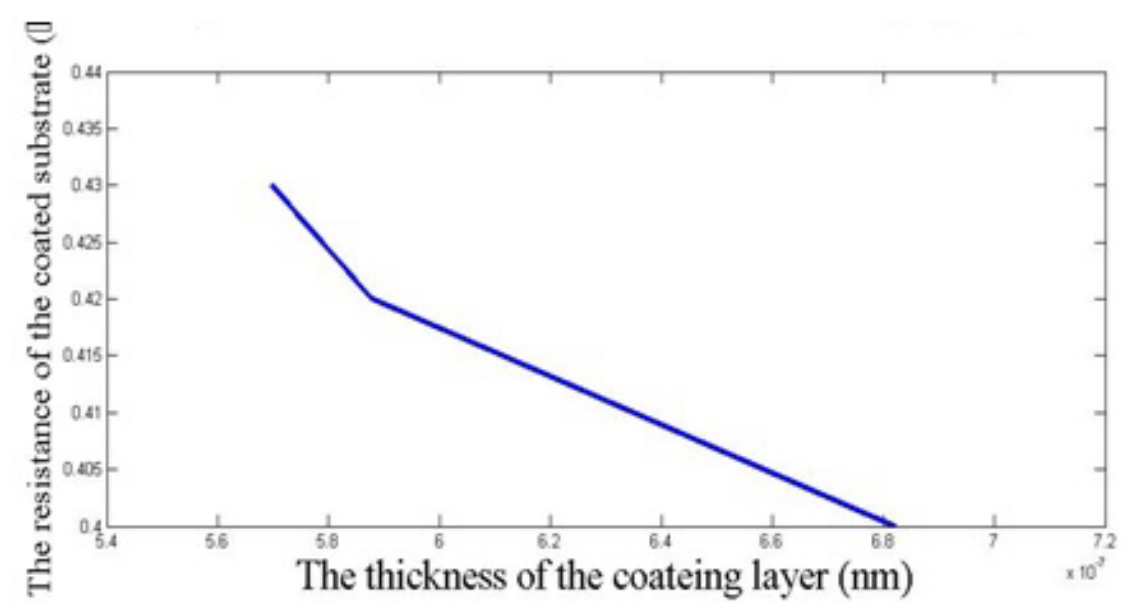

Fig. (8): The effect of thickness on the resistance of substrate for time of half hour 


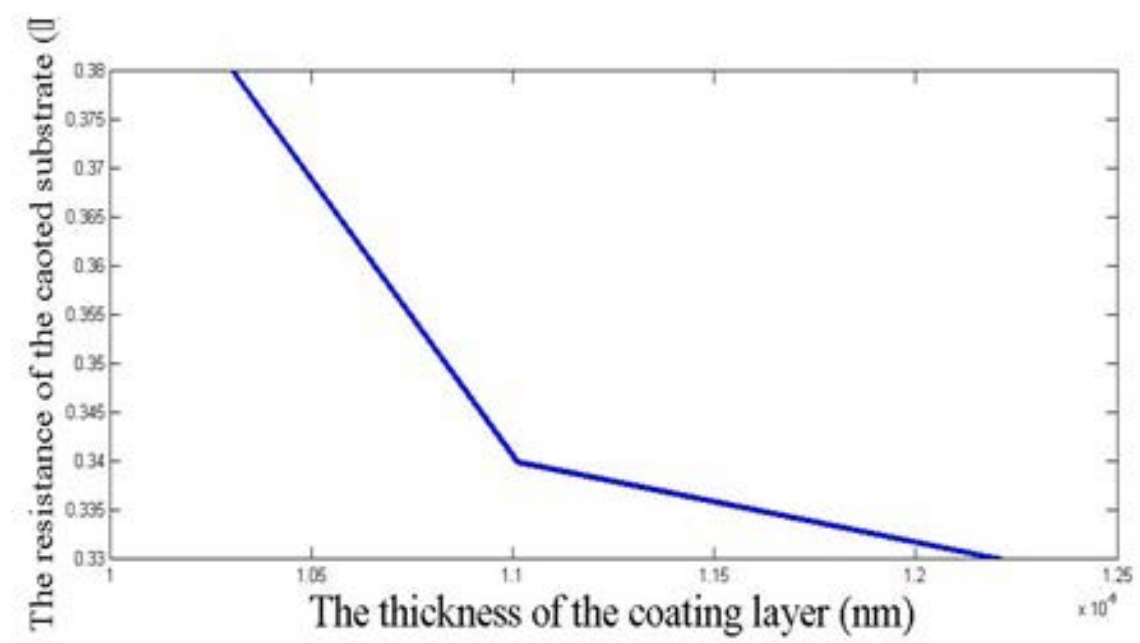

Fig. (9): The effect of thickness on the resistance of substrate for time of one hour

\section{Conclusions}

$\mathrm{Cu}$ films are deposited on $\mathrm{Al}$ substrates at various sputtering powers $(1.05,1.5,2 \mathrm{KW})$ using magnetron sputtering. The effect of sputtering power on the electrical and structural properties is investigated. It is obvious from the curves that by increasing the thickness of the coated layer for power from 1.05 to $1.5 \mathrm{KW}$, the resistance decreases by $2.32 \%$ for time of half hour and by $10.52 \%$ for time of one hour. After this power, there is nearly double decrease in the resistance by $4.76 \%$ for half hour coating and about $2.94 \%$ decreasing ratio for one hour coating. From this discussion, it is noticed that by applying the coating process for long time, but not for more MS power, the minimization of the resistance could be obtained. With decreasing the electrical resistance, the power transmitted in transmission system will be increased and losses will be decreased. Also, by increasing the MS applied power, the thickness of the coating layer increases and the resistance decreases.

\section{References}

1- Priya G. Deshmukh Prof. S.S. Katariya, "Nanotechnology Applications In The Energy Sector", International Journal of Advancements in Research \& Technology, Volume 2, Issue3, March2013 IISSN 2278-7763.

2- Kwang-Un Jeong, Jee Young Lim, Jong-Young Lee, Seong Lak Kang and Changwoon Nah, "Polymer nanocomposites reinforced with multiwalled carbon nanotubes for semiconducting layers of high-voltage power cables", Published online in Wiley Interscience: 12 November 2009.

3- Mahmoud A. EL Sabbagh, Samir M. El-Ghazaly and Hameed A. Naseem, "Carbon Nanotube-Based Planar Transmission Lines", IEEE, 2009, PP 353356.

4- Yao Zhao, Jinquan Wei1, Robert Vajtai1, Pulickel M. Ajayan1 \& Enrique V. Barrera1, "Iodine doped carbon nanotube cables exceeding specific electrical conductivity of metals", SCIENTIFIC REPORTS, September 2011, PP 1-83.

5- H.M. Mahmoud, M.A.Mehanna and S. K. Elsayed, "The Effectiveness of Carbon Nano Tube (CNT) in Voltage Stability and Power Transfer Capability", Nature and Science, 2012, 10 (4).

6- Minh-Tung Le;, Yong-Un Sohn, Jae-Won Lim; and Good-Sun Choi," Effect of Sputtering Power on the Nucleation and Growth of Cu Films Deposited by Magnetron Sputtering", Materials Transactions, Vol. 51, No. 1 (2010), pp. 116 to 120.

7- Arun Augustin, K. Rajendra Udupa, Udaya Bhat K.," Effect of Pre-Zinc Coating on the Properties and Structure of DC Magnetron Sputtered Copper Thin Film on Aluminium", American Journal of Materials Science 2015, Vol. 5, No.(3C), PP 58-61. 